\title{
Benchmarking in Norwegian Industry and Relationship Benchmarking
}

Bjørn Andersen, Msc.Eng./Ph.D.-student, Department of Production and Quality, Engineering, Norwegian Institute of Technology, N-7034 TRONDHEIM, Phone + 47735971 22, Fax + 47735971 17, e-mail bjorna@protek.unit.no

\section{BENCHMARKING IN NORWEGIAN INDUSTRY}

Benchmarking is probably at the moment one of the most popular management tool. Courses, conferences (as you can self witness today), books, papers, etc., are flooding the market. Benchmarking is hot and "in" right now, especially in the US. The interest in benchmarking is rapidly growing in Europe as well, although it has not reached the same extent as in the US. Still, the number of companies actually using benchmarking, either sporadically or on a regular basis, is fairly low. Some European branches of large, often multinational, corporations do benchmarking. A few other companies, sensitive to the latest trends, do as well. Otherwise, the propagation of active benchmarking is slow.

In Norway, the situation is probably even worse. Except a very few Norwegian subsidiaries or affiliates of larger corporations, there have been no reports of benchmarking being used. As a response to this situation, the TOPP Program, a Norwegian program aimed at improving the productivity of Norwegian technology industries, has started an effort to introduce benchmarking to Norwegian companies. The model TOPP has used for organizing and financing the promotion of benchmarking can be used to get benchmarking started in other countries, areas, industries, etc., and will be described in this paper.

\subsection{The TOPP program}

The TOPP Program is running from 1991 to 1995, with a budget of about NOK 200 millions, partly financed by the Norwegian Research Council and partly by the companies involved. So far, approximately fifty companies are "members" of the program. Research personnel from the Norwegian Institute of Technology, NTH, and SINTEF, the Foundation for Technical and Industrial Research, in cooperation with consultants within different areas, perform the different activities of the program.

These activities range from productivity analyses, research projects, education (10 Ph.D.'s and a master degree in technology management), networking between the companies, improvement projects, and projects aimed at developing or promoting different tools and techniques.

\subsection{The benchmarking project}

One project of the latter type is on benchmarking, mainly as a consequence of the situation described above, an immense lack of knowledge and competence about benchmarking, both in Norwegian companies as well as among consultants and academic 
institutions. The project was started in December 1993 and is supposed to run for one year, with a budget of NOK two millions.

The purpose of the project is twofold:

- To upgrade the TOPP companies' knowledge about and abilities to perform benchmarking.

- To upgrade the competence within benchmarking at NTH/SINTEF.

Three of the TOPP companies were invited to participate in the project, where two researchers from NTH/SINTEF, of whom I am one, represent TOPP. The two of us have read an extensive body of literature on benchmarking as well as taken courses arranged by the International Benchmarking Clearinghouse. TOPP covers all expenses of the two researchers, both hours and travels. The companies contribute with the direct man hours of those from the companies working on the project and the cost of their travels. In addition, we cooperate with Xerox Quality Solutions UK, for project planning and implementation support and practical guidance on specific problems. The Xerox support is also covered by TOPP.

All in all, the financial structure of the project is very beneficial for the companies, which receive an extensive amount of support in return for the work they put into the project.

\subsection{Project approach}

The project focus is to bring benchmarking into the companies by attacking a specific problem of current interest. This way, we avoid using benchmarking as a hammer just looking for a nail to punch. If benchmarking is perceived in such a way, chances are the companies would not give the project the necessary support. When benchmarking is used as a tool for solving problems identified as important by the companies themselves, the project is likely to get far higher priority.

Before starting the actual implementation of benchmarking, a foundation of basic knowledge had to be built. For this purpose, a Norwegian, very practical handbook in benchmarking was written, partly prior to and partly in parallel with the implementation activities. Based on the handbook, we gave short training sessions, which covered only the most basic principles of benchmarking. To avoid the feeling of being overdosed by a massive amount of information even before the project really started, further training was/is given throughout the project as the need arose/arises, i.e., on-the-job-training.

We use a six-step benchmarking process (Watson, 1992), covering the phases of planning, searching for partners, collecting information, analyzing, adapting best practice to our own organization, and recycling the study. To guide the three companies through a complete benchmarking study, we arrange quite frequent meetings, where we actively participate in the process as facilitators. Activities that need to be performed are split between us, TOPP, and the companies, so that we take on part of the workload.

At milestones, i.e., completion of phases, Xerox is brought in to review what has been done in the phase, and prepare the team for the next phase. At this point, one of the companies is in the partner visit stage of the project, having visited some partners and planning another visit. The others are still trying the complete the planning phase. By November this year, all three of them will have performed a complete benchmarking study. 


\subsection{Conclusions}

Naturally, these three will have learned the method of benchmarking. However, to ensure that also the rest of the TOPP companies gain from the project, the handbook will be made available to them. Any learned experiences throughout the project, i.e., both positive elements and things to avoid, will be incorporated into the book. To further boost the interest, a seminar will be arranged where the project is described and representatives from the companies put forward their experiences and obtained results from benchmarking. Some financing for other companies will probably also be made available, but not to such an extent as in this project.

It is still too early to evaluate whether the project has given any results, but we see that the companies involved in the project has gained thorough insight into the concept of benchmarking. Furthermore, as other companies hear of the project, reactions are they would want to participate themselves. At the end of the year, I think we will see that a large number of the TOPP companies have started using benchmarking. How much of this can be attributed to this project and how much is a result of a general increase in attention, is hard to estimate. Still, I think this model is useful for promoting benchmarking.

\section{RELATIONSHIP B ENCHMARKING}

\subsection{Types of benchmarking}

There are basically two criteria for defining different types of benchmarking - what is being benchmarked and who is being used as benchmarking partner. Dependent on what type of company is being used as benchmarking partner, three types of benchmarking are defined (Andersen and Pettersen, 1994):

- Internal benchmarking, where other units within the same corporation are used as partners.

- Competitive benchmarking, where direct competitors are used as partners.

- Generic benchmarking, where partners are chosen regardless of industry.

Each of these all displays some advantages and disadvantages. Both internal and generic benchmarking avoids the problems of sensitive information and the fear of upgrading a competitor that clouds competitive benchmarking. However, the incentives for companies asked to be generic benchmarking partners are not overwhelming. The partner will probably have the opportunity to learn something himself, but this has to be balanced against the time spent on the benchmarker. Although surprisingly many companies accept to be benchmarking partners, many benchmarkers still experience problems gaining access to partners. One answer to the lack of access to partners might be what we have called relationship benchmarking, i.e., using companies, that one already has relationships with, as benchmarking partners.

\subsection{Benchmarking against suppliers}

One of the three companies participating in the TOPP benchmarking project has chosen purchasing as the process to improve. Prior to the start of the benchmarking project, 
this company had already planned a visit to some of their suppliers to discuss improvements in the supplier-customer relationships. As this visit was already planned, we decided to include it in the benchmarking project, using the suppliers as benchmarking partners as well.

This way, the visit had two purposes; both to discuss and improve the interface between the two companies, e.g., more and better prognoses, EDI-ordering, etc., and to discuss their respective purchasing practices, in order to learn from each other.

Using suppliers, or any companies with which the benchmarker has already got an established relationship with, as benchmarking partners, does not directly fall within any of the earlier defined types of benchmarking. Viewing the whole supply chain as one superorganization, it has some similarities with internal benchmarking. As the other company might be in some totally different industry, it might be thought of as a variant of generic benchmarking. Still, none of these really match.

\subsection{Relationship benchmarking}

The first reaction of the TOPP company, when they realized some of the unique advantages and disadvantages of benchmarking their suppliers, was that this did not fit into any of the three types of benchmarking we had told them about at the start of the project. They saw it as a "new" type of benchmarking, which they liked to name relationship benchmarking. Relationship benchmarking can be defined as benchmarking against companies with which the benchmarker in advance of the benchmarking study already has established a relationship.

It is probably not in the interest of benchmarking, as a field, to explode the number of different types of benchmarking, nor the number of different available process models for performing benchmarking. One of the truly strong sides of benchmarking is its inherent simplicity. On the other side, the defined types of benchmarking should not prevent benchmarkers from considering using a particular group of companies as benchmarking partners.

Simply to make benchmarkers aware of the possibility of using companies they have already established relationships with as partners, I would like to describe some of the advantages and disadvantages of this approach.

Some advantages with relationship benchmarking are:

- Strategic partners, i.e., suppliers, customers, or horizontal partners, will usually benefit from improvements their partner makes. A supplier will benefit from having a more stable customer with higher sales, a customer will benefit from a more stable supplier with better performance, etc. Therefore, strategic partners will generally be willing to act as benchmarking partners, and will probably give the benchmarker high priority and easy access to information.

- As the two companies know each other in advance of the study, time is saved as general presentations are not necessary.

- As the companies are already involved, there will be no problems concerning sensitive information.

- During the benchmarking study, there are possibilities for directly improving the relationship between the two companies, as between the TOPP company and their suppliers. 
- As a consequence of acting as benchmarking partner, the partner, through increased attention to the process in question, can also make improvements. This both makes it more attractive to act as partner and, in turn, is beneficial for the benchmarker, as he gets a higher performing partner.

- Again, through acting as benchmarking partner, the partner will probably learn how to perform benchmarking himself, which can lead to improvements.

Some disadvantages are:

- When benchmarking a supplier, there is an obvious danger that the supplier will use the opportunity to "sell himself", i.e., devote far more time to presenting products and processes that work well.

- There is also a possibility that any strategic partner will want to appear better than he actually is, which will give false input into the benchmarking study.

- There is no guarantee that the benchmarker has any strategic partners that are sufficiently good to justify spending resources on using as a benchmarking partner.

- If the benchmarking partner is a horizontal partner, e.g., a partner in a joint venture, it is likely that this partner is or will be a competitor, in which case benchmarking can contribute to upgrading the partner.

- If the two companies are engaged in a customer-supplier relationship, there is a danger of revealing information that can impact price or delivery condition negotiations.

A conclusion is that strategic partners, if sufficiently good, might be a good choice of benchmarking partners, as they will generally be willing partners, which will benefit from improvements the benchmarker makes. The benchmarker should though be aware of the risk of biased information and time wasted on presentation outside the scope of the study. Still, keep in mind that strategic partners might make well-suited benchmarking partners, as well.

\section{REFERENCES}

Andersen, B. and Pettersen, P.G. (1994) The Basics of Benchmarking: What, When, Why, and How, Proceedings from the 1994 Pacific Conference on Manufacturing, Djakarta, Indonesia

Watson, G.H. (1992) The Benchmarking Workbook: A dapting Best Practices for Performance Improvement, Productivity Press, Cambridge, MA 